\title{
Scattering mechanism in a step-modulated subwavelength metal slit: a multi-mode multi-reflection analysis
}

\author{
Chao $\mathrm{Li}^{1,2}$, Yun-Song Zhou ${ }^{2, \dagger}$, Huai-Yu Wang ${ }^{3, \ddagger}$, and Jian-Hong Guo ${ }^{2}$ \\ 1 School of Mathematical Sciences, Capital Normal University, Beijing 100048 China \\ 2 Center of Theoretical Physics, Department of Physics, \\ Capital Normal University. Beijing 100048, China and \\ 3 Department of Physics, Tsinghua University, Beijing 100084, China
}

(Dated: August 13, 2018)

\begin{abstract}
In this paper, the scattering/transmission inside a step-modulated subwavelength metal slit is investigated in detail. We firstly investigate the scattering in a junction structure by two types of structural changes. The variation of transmission and reflection coefficients depending on structural parameters are analyzed. Then a multi-mode multi-reflection model based on ray theory is proposed to illustrate the transmission in the step-modulated slit explicitly. The key parts of this model are the multi-mode excitation and the superposition procedure of the scatterings from all possible modes, which represent the interference and energy transfer happened at interfaces. The method we use is an improved modal expansion method (MEM), which is a more practical and efficient version compared with the previous one [Opt. Express 19, 10073 (2011)]. In addition, some commonly used methods, FDTD, scattering matrix method, and improved characteristic impedance method, are compared with MEM to highlight the preciseness of these methods.

PACS numbers: 78.68.+m, 78.20.-e, 42.79.Gn, 73.20.Mf
\end{abstract}




\section{INTRODUCTION}

Subwavelength metal slits, as a kind of metal/insulator/metal waveguides, have attracted much attention in recent years not only because of their ability to guide light beyond the diffraction limit, but also because of several remarkable advantages, such as strong field localization, simplicity, and convenience for fabrication and integration into optical circuits [1-14]. When light (infrared, visible spectrum) propagates along a metal/air interface, it will excite a collective oscillation of free electrons at the surface of the metal, causing a field exponentially decaying away from the interface. This mode is called as surface plasmon polariton (SPP) [2-6]. In a subwavelength metal/air/metal slit, the case is somehow different, since the in-slit SPP [5] wave decays exponentially in the metals and is flat in the air, which is the lowest eigenmode in the slit structure and the core part of the subwavelength metallic optics.

Step modulation is one of the key elements in photonic engineering that are employed in subwavelength metal structures to design and fabricate functional plasmonic devices, such as filters [7-10], reflectors [11], and photonic bandgap structures [11,12]. Besides, the step modulation is of important theoretical significance since they are helpful for investigating SPP scattering. Had the knowledge and combined with the staircase approximation and transfer matrix technique, numerical results of more complicated structures can be obtained.

Up to now, a number of methods have been used to calculate the SPP scattering/transmission inside a step-modulated slit. The finite-difference time-domain method (FDTD) is a well developed simulation method that provides relatively accurate results and has been considered as a standard for testing other theoretical methods [5-14]. The effective index method, on the other hand, is a simplified and direct theoretical method where only the SPP modes are involved in the calculation, a method of one mode approximation; how-

ever, this simplification causes the loss of the scattering information considerably and the numerical imprecision turned out to be considerable under some conditions [6]. Matsuzaki et al. presented a transmission model and gave a better description of the SPP scattering using the characteristic impedance method [13]. Pannipitiya et al. [14] suggested an improved version of this method, which will be called as improved characteristic impedance method (ICIM) in this paper. Lin et al. presented a similar transmission model and used the scattering matrix method (SMM) to calculate the transmission [7-9]. Although the cal- 
culated results from these two methods fit the FDTD results, two approximations, one mode approximation and quasi-statistic approximation, are used in the calculation, which limits their application scope and numerical precision, as will be discussed in Sec. 3 below. Recently, we successfully applied the modal expansion method (MEM) in discussing the wave behavior inside a symmetric step-modulated slit [6]. This method did not involve the two mentioned approximations and provided more accurate results.

In this paper, the MEM is further improved so as to apply to the asymmetric modulated case in investigating the scattering/transmission mechanisms inside a step-modulated slit. A multi-mode multi-reflection model is proposed to explain the transmission process. A remarkable advantage of MEM is that its precision is controllable. This enables us to discuss the preciseness of FDTD and ICIM by comparing the results from these methods and MEM.

The paper is arranged as follows. Section 2 sets our model of a step-modulated metal slit and presents the improved MEM formulas. In Sec. 3, the scattering in a junction structure is studied firstly as a prerequisite for later discussion, and then a multi-mode multi-reflection model is proposed to reveal the transmission mechanism in a step-modulated slit. Comparisons between MEM, FDTD, SMM, and ICIM are also given in this section to highlight the restriction of the one mode approximation and quasi-statistic approximation. Finally, conclusions are presented in Sec. 4.

\section{MODEL AND THE IMPROVED MEM}

In this section, we set the model of single-slit structure and present the formulas of the improved MEM which is more practical and efficient compared with that in our previous work [6].

The model structure is shown in Fig. 1. It is infinitely large in $y z$ plane but confined in $x$ direction by perfectly conducting walls at $x=0$ and $x=L$. The structure is divided into three regions along $x$ direction, composed of silver/air/silver, and three layers along $y$ direction. The lower boundary of the $l$ th layer is labeled as $Q^{(l-1)}$, and the interfaces between regions are by $x_{1}^{(l)}$ and $x_{2}^{(l)}$. The denotations $q^{(l)}$ and $w^{(l)}$ label layer height and slit width, respectively. A transverse magnetic (TM) wave, with magnetic field $\mathbf{H}$ being in $z$ direction, is normally launched from $y=Q^{(0)}$ in Layer 1 and propagates upward. The structural parameters are given in the caption of Fig. 1. 


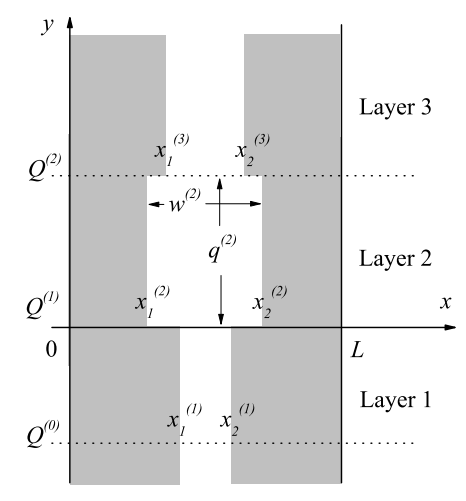

FIG. 1: Sketch of a step-modulated metal slit structure confined in $x$ direction with perfectly conducting walls at 0 and $L=2 \mu \mathrm{m}$. A TM wave with wavelength $\lambda_{0}$ is normally launched at $y=Q^{(0)} \cdot Q^{(1)}=0$.

The dielectric constant of silver as a function of the wavelength of the incident wave $\lambda_{0}$ is evaluated as $\varepsilon_{A g}=\left(3.57-54.33 \lambda_{0}^{2}\right)+i\left(-0.083 \lambda_{0}+0.921 \lambda_{0}^{3}\right)$ by fitting the experimental data [15], which is valid for $0.6 \leq \lambda_{0} \leq 1.6 \mu \mathrm{m}$. In this paper, the wavelength is mainly set as $\lambda_{0}=1 \mu \mathrm{m}$; thus, $\varepsilon_{A g}=-50.76+0.083 i$.

In the remaining part of this section, we suggest an improved version of the MEM, which has the same output as the previous one [6] but is easier and faster.

The substance of MEM is to expand the unknown functions (electromagnetic field distribution in present case) by a complete set of orthogonal functions. This makes the MEM has two folds: one is the eigenvalue problem of the system; the other is to establish and solve the coupled equations subject to boundary conditions. However, the choice of the complete set in modal expansion is not unique, but depends on the configuration of the system. It can be the eigenfunctions of a specific structure or other functions such as sine or exponential functions.

In Ref. [6], the fields in the given structure were handled by separation of variables. The factors containing $x$ variable were expanded by the eigenmodes $\left\{\psi_{n}^{(l)}(x)\right\}$ between two perfectly conducting walls. The magnetic fields were expressed as

$$
H_{z}(x, y)= \begin{cases}\sum_{n} \psi_{n}^{(1)}(x)\left[I_{n} e^{i k_{y n}^{(1)}\left(y-Q^{(0)}\right)}+R_{n} e^{-i k_{y n}^{(1)}\left(y-Q^{(1)}\right)}\right], & Q^{(0)} \leq y<Q^{(1)} \\ \sum_{n} \psi_{n}^{(2)}(x)\left[E_{n} e^{i k_{y n}^{(2)}\left(y-Q^{(1)}\right)}+F_{n} e^{-i k_{y n}^{(2)}\left(y-Q^{(2)}\right)}\right], & Q^{(1)} \leq y<Q^{(2)} \\ \sum_{n} \psi_{n}^{(3)}(x) T_{n} e^{i k_{y n}^{(3)}\left(y-Q^{(2)}\right)}, & Q^{(2)} \leq y<\infty,\end{cases}
$$


where $I_{n}, R_{n}, E_{n}, F_{n}$, and $T_{n}$ were expansion coefficients which involved the scattering/transmission information of every eigenmode. It was inevitable to solve a transcendental equation in order to achieve the eigenvalues and eigenfunctions. Even with assistance of a powerful root-seeking method [16], this procedure was still time-consuming. Moreover, each layer had its own eigenfunctions. At an interface, it was required by the boundary conditions to calculate the overlap between the eigenfunctions at the two sides of the interface, called as coupling integrals. Such integrals brought complexity to the program.

To avoid these difficulties, in this paper the factors containing $x$ variable were expanded by a sine basis subject to the perfectly conducting boundary condition. That is to say, the complete set $\left\{\varphi_{n}(x)\right\}$ is chosen as

$$
\varphi_{n}(x)=\sqrt{2 / L} \sin \left(k_{x n} x\right), \quad k_{x n}=n \pi / L, \quad n=1,2,3 \cdots
$$

The eigenvalues $k_{x n}$ are solely determined by the distance between the two perfectly conducting walls, independent of the positions $x_{1}^{(l)}$ and $x_{2}^{(l)}$, so that is valid for all the three layers.

Correspondingly, the magnetic fields and its derivative in the three layers can be expressed as $[17]$

$$
H_{z}(x, y)= \begin{cases}\sum_{n} \varphi_{n}(x) \sum_{m} W_{n, m}^{(1)}\left[i_{m} e^{i k_{y m}^{(1)}\left(y-Q^{(0)}\right)}+r_{m} e^{-i k_{y m}^{(1)}\left(y-Q^{(1)}\right)}\right], & Q^{(0)} \leq y<Q^{(1)} \\ \sum_{n} \varphi_{n}(x) \sum_{m} W_{n, m}^{(2)}\left[e_{m} e^{i k_{y m}^{(2)}\left(y-Q^{(1)}\right)}+f_{m} e^{-i k_{y m}^{(2)}\left(y-Q^{(2)}\right)}\right], & Q^{(1)} \leq y<Q^{(2)} \\ \sum_{n} \varphi_{n}(x) \sum_{m} W_{n, m}^{(3)} t_{m} e^{i k_{y m}^{(3)}\left(y-Q^{(2)}\right)}, & Q^{(2)} \leq y<\infty\end{cases}
$$

where $i_{m}, r_{m}, e_{m}, f_{m}$, and $t_{m}$ are the expansion coefficients. The insertions of Eq. (3) into Helmholtz equation yields an eigenvalue problem in each layer expressed by $A^{(l)} W^{(l)}=$ $\left(i k_{y}^{(l)}\right)^{2} W^{(l)}$ with $i k_{y}^{(l)}$ and $W^{(l)}$ being the eigenvalues and eigenfunctions, and the operator $A^{(l)}$ being [18]

$$
A^{(l)}=-\left[\tilde{E}^{(l)}\right]^{-1}\left\{k_{0}^{2}[I]+[K]\left[E^{(l)}\right]^{-1}[K]\right\}
$$

where

$$
\left\{\begin{array}{l}
{\left[\tilde{E}^{(l)}\right]_{m n}=\int_{0}^{L} \varphi_{m}(x) \varphi_{n}(x) / \varepsilon^{(l)}(x) d x} \\
{\left[E^{(l)}\right]_{m n}=\int_{0}^{L} \varphi_{m}(x) \varphi_{n}(x) \varepsilon^{(l)}(x) d x} \\
{[K]_{m n}=\int_{0}^{L} \varphi_{m}(x) \frac{\partial}{\partial x} \varphi_{n}(x) d x} \\
{[I]_{m n}=\delta_{m n} .}
\end{array}\right.
$$


In these equations, $[\cdot]$ denotes a $N \times N$ matrix where $N$ is the truncation number, and $k_{0}=2 \pi / \lambda_{0}$ is the wave vector in vacuum.

Here we mention the two advantages of the sine expansion in $x$ direction. One is that the eigenvalue problem, Eq. (4), is very easy for computer implementation, which avoids the cumbersome solution-seeking procedure necessary in the eigenmode expansion [6]. The other is that the complete set of sine functions are the same for all layers, so that the coupling integrals at the interfaces become quite simple. These two advantages make the calculation program greatly simplified.

Corresponding to Eq. (3), the derivative of the magnetic field is expressed as

$$
\frac{1}{\varepsilon} \frac{\partial}{\partial y} H_{z}(x, y)= \begin{cases}\sum_{n} \frac{\varphi_{n}(x)}{\varepsilon^{(1)}} \sum_{m} W_{n, m}^{(1)} i k_{y m}^{(1)}\left[i_{m} e^{i k_{y m}^{(1)}\left(y-Q^{(0)}\right)}-r_{m} e^{-i k_{y m}^{(1)}\left(y-Q^{(1)}\right)}\right], & Q^{(0)} \leq y<Q^{(1)} \\ \sum_{n} \frac{\varphi_{n}(x)}{\varepsilon^{(2)}} \sum_{m} W_{n, m}^{(2)} i k_{y m}^{(2)}\left[e_{m} e^{i k_{y m}^{(2)}\left(y-Q^{(1)}\right)}-f_{m} e^{-i k_{y m}^{(2)}\left(y-Q^{(2)}\right)}\right], & Q^{(1)} \leq y<Q^{(2)} \\ \sum_{n} \frac{\varphi_{n}(x)}{\varepsilon^{(3)}} \sum_{m} W_{n, m}^{(3)} i k_{y m}^{(3)} t_{m} e^{i k_{y m}^{(3)}\left(y-Q^{(2)}\right)}, & Q^{(2)} \leq y<\infty,\end{cases}
$$

Applying the layer boundary conditions, we obtain the coupled equations as follows:

$$
\left\{\begin{array}{l}
\sum_{m} W_{p m}^{(1)}\left[i_{m} e^{i k_{y m}^{(1)} q^{(1)}}+r_{m}\right]=\sum_{m} W_{p m}^{(2)}\left[e_{m}+f_{m} e^{i k_{y m}^{(2)} q^{(2)}}\right] \\
\sum_{n} \tilde{E}_{p n}^{(1)} \sum_{m} W_{n m}^{(1)} k_{y m}^{(1)}\left[i_{m} e^{i k_{y m}^{(1)} q^{(1)}}-r_{m}\right]=\sum_{n} \tilde{E}_{p n}^{(2)} \sum_{m} W_{n m}^{(2)} k_{y m}^{(2)}\left[e_{m}-f_{m} e^{i k_{y m}^{(2)} q^{(2)}}\right] \\
\sum_{m} W_{p m}^{(2)}\left[e_{m} e^{i k_{y m}^{(2)} q^{(2)}}+f_{m}\right]=\sum_{m} W_{p m}^{(3)} t_{m} \\
\sum_{n} \tilde{E}_{p n}^{(2)} \sum_{m} W_{n m}^{(2)} k_{y m}^{(2)}\left[e_{m} e^{i k_{y m}^{(2)} q^{(2)}}-f_{m}\right]=\sum_{n} \tilde{E}_{p n}^{(3)} \sum_{m} W_{n m}^{(3)} k_{y m}^{(3)} t_{m},
\end{array}\right.
$$

The incident coefficients $i_{m}$ are determined by the incident wave. In this paper, the incident wave is always a SPP wave launched in Layer 1 , namely, $\psi_{1}^{(1)}(x)$. Therefore, one naturally has

$$
\int_{0}^{L} \varphi_{p}(x) \psi_{1}^{(1)}(x) d x=\sum_{m} W_{p m}^{(1)} i_{m}
$$

which determines the coefficients $i_{m}$. After setting the incident coefficients $i_{m}$, the four groups of coefficients, $r_{m}, e_{m}, f_{m}$, and $t_{m}$, can be obtain from Eq. (7). Thus all the field quantities are obtained.

In this paper, we will focus on discussing the reflection/transmission mechanisms, which are mainly presented by the reflection coefficients $R_{n}$ and transmission coefficients $T_{n}$ in Eq. (1). For example, the amplitudes of the SPP modes in Layer 1 and $3,\left|R_{n}\right|$ and $\left|T_{n}\right|$, are the reflection and transmission efficiencies of the system, and their arguments, $\arg \left(R_{n}\right)$ and $\arg \left(T_{n}\right)$, are the corresponding phase shifts. Therefore, a projection between the fields 
calculated by Eq. (3) and the eigenmodes $\left\{\psi_{n}^{(l)}(x)\right\}$ is implemented for obtaining the $R_{n}$ and $T_{n}$. In the following, the absolute values of these coefficients may generally be named as excitation efficiency.

Thus we accomplish our formulation presentation. This method is briefly outlined as follows: the field is expanded by sine functions which are complete and uniform in all layers. The corresponding eigenvalue problem becomes a matrix form as shown in Eq. (4), which makes the calculation quite easy. Accordingly, the procedure here is much more practical and efficient compared to the previous one [6]. At last, the overlaps between the calculated field and the eigenmodes in Eq. (1) give the required reflection and transmission coefficients necessary for physical analysis.

Although we merely study the three-layer structure, our procedure developed here is easily applied to more complicated structures by implanting the S matrix algorithm [19] or the enhanced transmittance matrix approach [20].

\section{NUMERICAL RESULTS AND ANALYSIS}

In this section, we investigate the scattering/transmission mechanisms inside a stepmodulated subwavelength metal slit. To do so, the scattering in a junction structure is first discussed in detail, since the slit comprises more than one junction structure. Then we disclose the multi-mode multi-reflection model in the transmission process in the slit. By the way, the numerical precision of FDTD and ICIM is discussed by comparing results of these two methods and MEM.

In calculation, the confinement is set as $L=2 \mu \mathrm{m}$. We have tested that 800 modes, $N=800$ in Eq. (4), are enough to give results with precision up to four significant digits. The convergence test for truncation number $N$ and the preciseness test for confined width $L$ will be carried out later in Fig. 7 .

\section{A. Junction structures}

A junction structure is the connection of two half-infinitely long slits with widths being denoted by $w^{(1)}$ and $w^{(3)}$, respectively, which can be easily realized in Fig. 1 by setting the height of Layer 2 to 0 . In Ref. [6], some scattering properties of symmetric structures have 
been revealed. For example, the main ingredient of the fields inside the slits were guided modes which played a very important role in scattering/transmission, and the unimportant components were the radiation modes excited which were necessary to fulfill boundary condition, but had little contribution to the transmission. So the following discussion will focus on the guided modes. However, the discussion in Ref. [6] was limited to the symmetric case. Here we present a detailed investigation on how the scattering is affected by asymmetry.

Two types of structural changes are considered. In Type I, the widths of the two slits are fixed and the position of the narrower one can be anywhere between left to right, as shown in the inner panel of Figs. 2(a) and (c). In Type II, the left walls of the slits are aligned and the width of the narrower one is fixed, but that of the wider one can vary, as schematically shown in the inner panel of Figs. 3(a) and (c).

The results of Type I structure are plotted in Fig. 2. The left wall of the wider slit is at $x_{1}=0.6 \mu \mathrm{m}$. For $w^{(1)}=0.1$ and $w^{(3)}=0.8 \mu \mathrm{m}$, when the position of narrower slit is moved from the left to right, the excitation efficiencies and their phase shifts are plotted in Figs. 2(a) and (b), respectively. In Figs. 2(c) and (d) are the excitation efficiencies and their phase shifts of structure $w^{(1)}=0.8$ and $w^{(3)}=0.1 \mu \mathrm{m}$. In Figs. 2(e) and (f) are the absolute values of the first three eigenfunctions of the narrower and wider slits, respectively. In Figs. 2(a), (c), (e) and (f) the absolute values are plotted because these quantities are complex. The eigenmodes in the narrower and wider slits are denoted by $\psi_{n}^{(n a)}$ and $\psi_{n}^{(w i)}$, respectively. In Figs. 2(e) and (f), the lowest modes $\psi_{1}^{(n a)}$ and $\psi_{1}^{(w i)}$, plotted by the black curves, are just SPP modes, and the second modes $\psi_{2}^{(n a)}$ and $\psi_{2}^{(w i)}$, plotted by the red curves, are of actually antisymmetric wave functions within the slits. Note that the curve of $\left|\psi_{3}^{(w i)}\right|$ is divided into three parts by two zeros. The sign of the central part of $\psi_{3}^{(w i)}$ is contrary to the other parts.

We notice that under our present parameters, only the first two eigenmodes of the narrower slit $\psi_{1}^{(n a)}$ and $\psi_{2}^{(n a)}$ are within the slit (guided modes), see the black and red lines in Fig. 2(e). The wave functions of the higher modes mainly distribute within the metal (radiation modes), see, as an example, the third mode $\psi_{3}^{(n a)}$ in Fig. 2(e). The behavior of $\left|\psi_{3}^{(n a)}\right|$ with the position of the narrower slit has to be explicitly given as following. When the slit moves rightwards, the width of metal at the right side of the slit becomes thinner, so that the hill is compressed. If the narrower slit is on the right side of the center position $x=1 \mu \mathrm{m}$, the hill will appear at the left side of the slit. While if the slit is just at or very near the center $x=1 \mu \mathrm{m}$, there will be two hills at the two sides of the slit, respectively, 

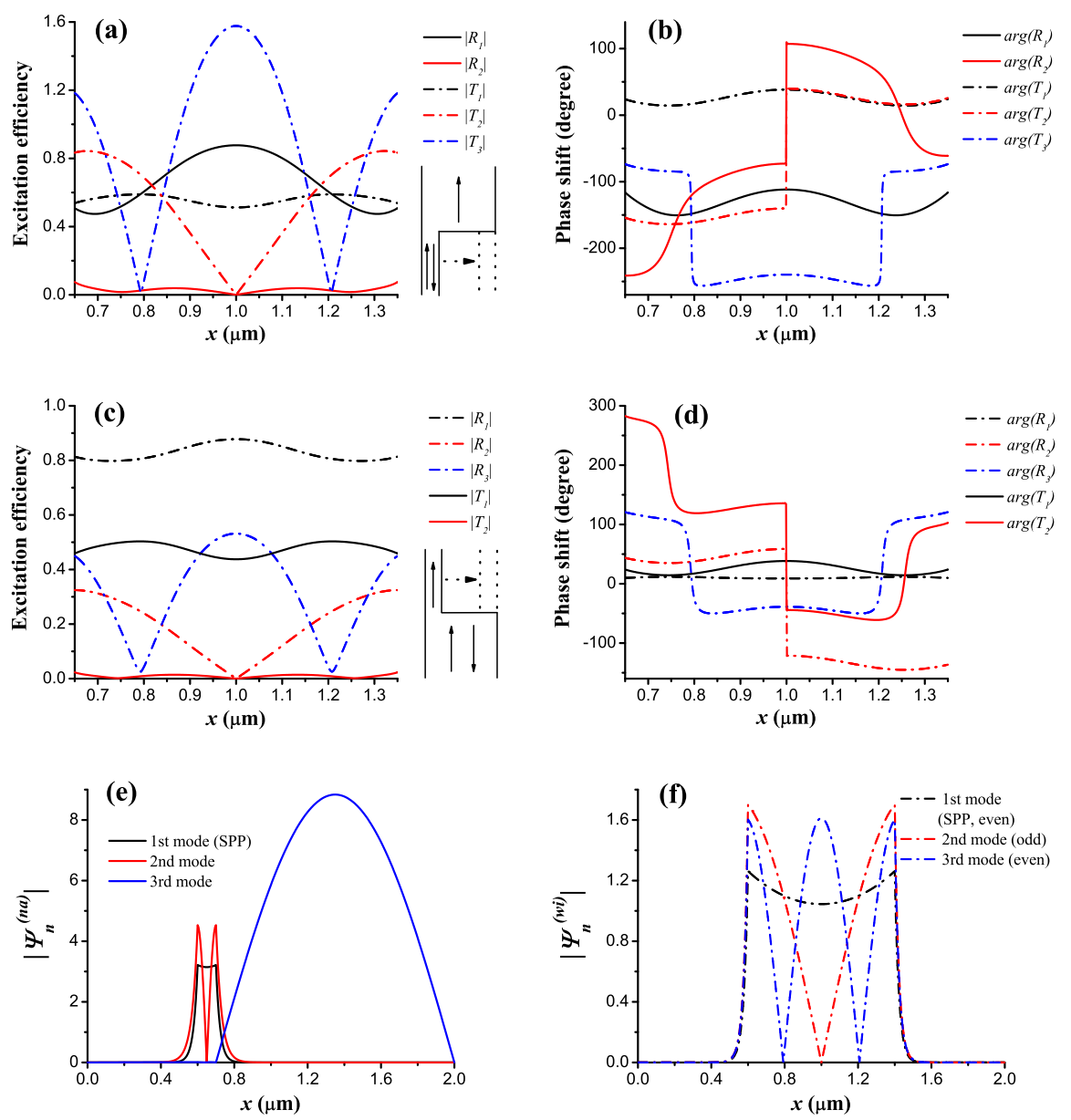

FIG. 2: (color online). Scattering in Type I junction structures. The left wall of the wider slit is fixed at $x_{1}=0.6 \mu \mathrm{m} . Q^{(0)}=Q^{(1)}=Q^{(2)}=0$. In all the figures, the results of the wider slit are plotted by solid lines and those of the narrower slit by dash-dotted lines. The narrower slit moves from the left to right. In Figs. (a) to (d), the $x$-axes are its central position. For the structure with $w^{(1)}=0.1$ and $w^{(3)}=0.8 \mu \mathrm{m}$, (a) excitation efficiency; (b) phase shift. For the structure with $w^{(1)}=0.8$ and $w^{(3)}=0.1 \mu \mathrm{m}$, (c) excitation efficiency; (d) phase shift. In (e) and (f) plotted are the absolute value of the eigen functions in the narrower and wider slits, respectively, when their left walls are aligned.

since the structure in this case is symmetric [6].

Three obvious features of excitation efficiencies can be seen in Figs. 2(a) and (c). The first is that all the curves there exhibit a central symmetry, because all the configurations are symmetric with respect to the central line at $x=1 \mu \mathrm{m}$. The second is, from comparison 
of the black and red solid lines in Figs. 2(a) and (c), that the excitation efficiencies of the SPP modes in narrow slits are much larger than those of the second modes. The third is, by inspection of dash-dotted lines in Figs. 2(a) and (c), that the shapes of the efficiency curves of the modes in wider slits resemble their eigenfunctions $\left|\psi_{n}^{(w i)}\right|$ in Fig. 2(f). The latter two features can be attributed to the treatment of MEM which involves a mutual expansion between the modes in different layers.

We should keep in mind that the total field at the layer boundary, $H_{z}(x)$, can be respectively achieved by the linear combination of eigenfunctions in the narrower and wider slit, and the expansion coefficients depend on the position of the narrower slit, subject to boundary conditions. Then, the curves in Figs. 2(a) and (c) can be explained qualitatively.

We first see the case where the wave is incident from the narrower slit to wider one, as shown by the inset in Fig. 2(a). For reflected waves, the reflection efficiencies $\left|R_{n}\right|$ are proportional to the projection $\int_{0}^{L} \psi_{n}^{(n a)}(x) H_{z}(x) d x$ where the integration is along the interface between the narrower and wider slits. Since the eigenfunctions in the wider slit or their combination, $H_{z}(x)$, can be seen as a smooth variation within the range of narrower slit, the reflection efficiency of the SPP mode in the narrower slit, $\left|R_{1}\right| \propto \int_{0}^{L} \psi_{1}^{(n a)}(x) H_{z}(x) d x \approx$ $\int_{w^{(n a)}} \psi_{1}^{(n a)}(x) H_{z}(x) d x$, is dominant because its eigenfunction is also smooth within the slit, and that $\left|R_{2}\right| \propto \int_{0}^{L} \psi_{2}^{(n a)}(x) H_{z}(x) d x \approx \int_{w(n a)} \psi_{2}^{(n a)}(x) H_{z}(x) d x$ is very small because the second mode is an antisymmetric function within the slit. For the transmitted waves, the transmission efficiencies $\left|T_{n}\right|$ are qualitatively determined by $\int_{0}^{L} \psi_{n}^{(w i)}(x) H_{z}(x) d x$, which includes $\int_{0}^{L} \psi_{n}^{(w i)}(x) \psi_{1}^{(n a)}(x) d x, \int_{0}^{L} \psi_{n}^{(w i)}(x) \psi_{2}^{(n a)}(x) d x, \int_{0}^{L} \psi_{n}^{(w i)}(x) \psi_{3}^{(n a)}(x) d x$, and so on. We have already known that the excitation of the second mode in the narrow slit is very small, so that the contribution of the factor $\int_{0}^{L} \psi_{n}^{(w i)}(x) \psi_{2}^{(n a)}(x) d x$ is negligible. The contribution of the radiation modes is relatively complicated, but unimportant because what happened inside the slit is the key part of the scattering procedure; while the radiation modes localized in metal are excited to fulfill the boundary condition outside the slit. That is why we try to avoid theses modes in the discussion. By several numerical tests, it is sure that the radiation modes do have contribution to the transmitted waves but the contribution is comparatively small. Therefore, the factor $\int_{0}^{L} \psi_{n}^{(w i)}(x) \psi_{1}^{(n a)}(x) d x \approx \int_{w^{(n a)}} \psi_{n}^{(w i)}(x) \psi_{1}^{(n a)}(x) d x$ mainly determines the transmission efficiencies $\left|T_{n}\right|$. As an example, let us see the $\left|T_{3}\right|$ curve. $\left|T_{3}\right| \propto \int_{w^{(n a)}} \psi_{3}^{(w i)}(x) \psi_{1}^{(n a)}(x) d x$, where $\psi_{1}^{(n a)}$ is smooth within a narrow region, see, the black line in Fig. 2. When the narrower slit is positioned at the left side with its center being 
at $x=0.65 \mu \mathrm{m},\left|\psi_{3}^{(w i)}\right|$ has a maximum at this position. Therefore the projection of $\psi_{1}^{(n a)}$ onto $\psi_{3}^{(w i)}$ is at a maximum. As the narrower slit moves rightwards, we image that the black curve in Fig. 2(e) shifts rightwards. At $x=0.794 \mu \mathrm{m},\left|\psi_{3}^{(w i)}\right|$ is zero. Accordingly, the projection of $\psi_{1}^{(n a)}$ at this position onto $\psi_{3}^{(w i)}$, as well as $\left|T_{3}\right|$, reaches zero. Between $x=0.65$ and $x=0.794 \mu \mathrm{m},\left|T_{3}\right|$ should drop from the maximum to zero. We notice that around the zero, the phase of $T_{3}$ changes nearly $\pi$. At the other zero of $\left|\psi_{3}^{(w i)}\right|$ at $x=1.206 \mu \mathrm{m},\left|T_{3}\right|$ again reaches zero and its phase changes nearly $\pi$ once more. This analysis explains why the shape of $\left|T_{3}\right|$ is like to $\left|\psi_{3}^{(w i)}\right|$. It is the narrow and smooth profile of $\left|\psi_{1}^{(n a)}\right|$ that causes the similarity of the curves between $\left|T_{3}\right|$ and $\left|\psi_{3}^{(w i)}\right|$ curves. The $\left|T_{2}\right|$ curve in Fig. 2(a) is understood in the same way. $\left|T_{1}\right|$ is mainly determined by $\int_{w^{(n a)}} \psi_{1}^{(w i)}(x) \psi_{1}^{(n a)}(x) d x$, which is a smooth and relatively flat curve due to the smooth variations of both SPP waves.

We next see the case where a SPP wave is incident from the wider slit to narrower one, as shown in the inset of Fig. 2(c), the incident wave being a smooth curve within the range of the wider slit width, see the black curve in Fig. 2(f). We again begin with the waves in narrower slit. $\left|T_{n}\right|$ is proportional to the integral $\int_{0}^{L} \psi_{n}^{(n a)}(x) H_{z}(x) d x$, where $H_{z}(x)$ is the combination of eigenfunctions in the wider slit and considered as a smooth varying curve within the range of the narrower slit, so that the transmission efficiency of the SPP mode is dominant and much larger than that of the second mode. For the waves in the wilder slit, ignoring the contribution of the second mode and radiation modes, the reflection efficiencies $\left|R_{n}\right|$ is mainly determined by $\int_{w^{(n a)}} \psi_{n}^{(w i)}(x) \psi_{1}^{(n a)}(x) d x$, leading to the fact that the $\left|R_{n}\right|$ curves in Fig. 2(c) have similar shapes as $\left|T_{n}\right|$ curves in Fig. 2(a).

The transmission efficiency $\left|T_{1}\right|$ in Fig. 2(a) is exactly the same as that in Fig. 2(c), and the efficiencies $\left|R_{1}\right|,\left|T_{2}\right|$ and $\left|T_{3}\right|$ in Fig. 2(a) have the same behavior as $\left|R_{1}\right|,\left|R_{2}\right|$ and $\left|R_{3}\right|$ in Fig. 2(c), respectively, although with different values. The reflection efficiencies $\left|R_{1}\right|$ and $\left|R_{2}\right|$ in Fig. 2(c) are higher than those in Fig. 2(a). This is because Fig. 2(c) represents the case that wave incident from a wider slit to a narrower one, which needs to squeeze light into a narrower space, so the higher reflection is understandable.

The variations of the scattering phase shifts for the above two different incident cases are plotted in Fig. 2(b) and (d). It is seen that the phases of both $T_{1}$ in these two figures are also the same. We note that at the positions where $\psi_{n}^{(w i)}$ is zero, the corresponding coefficients $R_{n}$ and $T_{n}$ have phase change of $\pi$.

The results of Type II structure are plotted in Fig. 3. The left walls of the slits are 
aligned at $x_{1}=0.6 \mu \mathrm{m}$. The width of the narrower slit is $0.1 \mu \mathrm{m}$, but that of the wider one, denoted by $w$, varies from 0.1 to $0.8 \mu \mathrm{m}$, as shown in the insets in Figs. 3(a) and (c). The most distinct feature is the drastic changes of the excitation efficiencies over a narrow range of slit width, as shown in Figs. 3(a) and (c) near $w=0.46 \mu \mathrm{m}$.
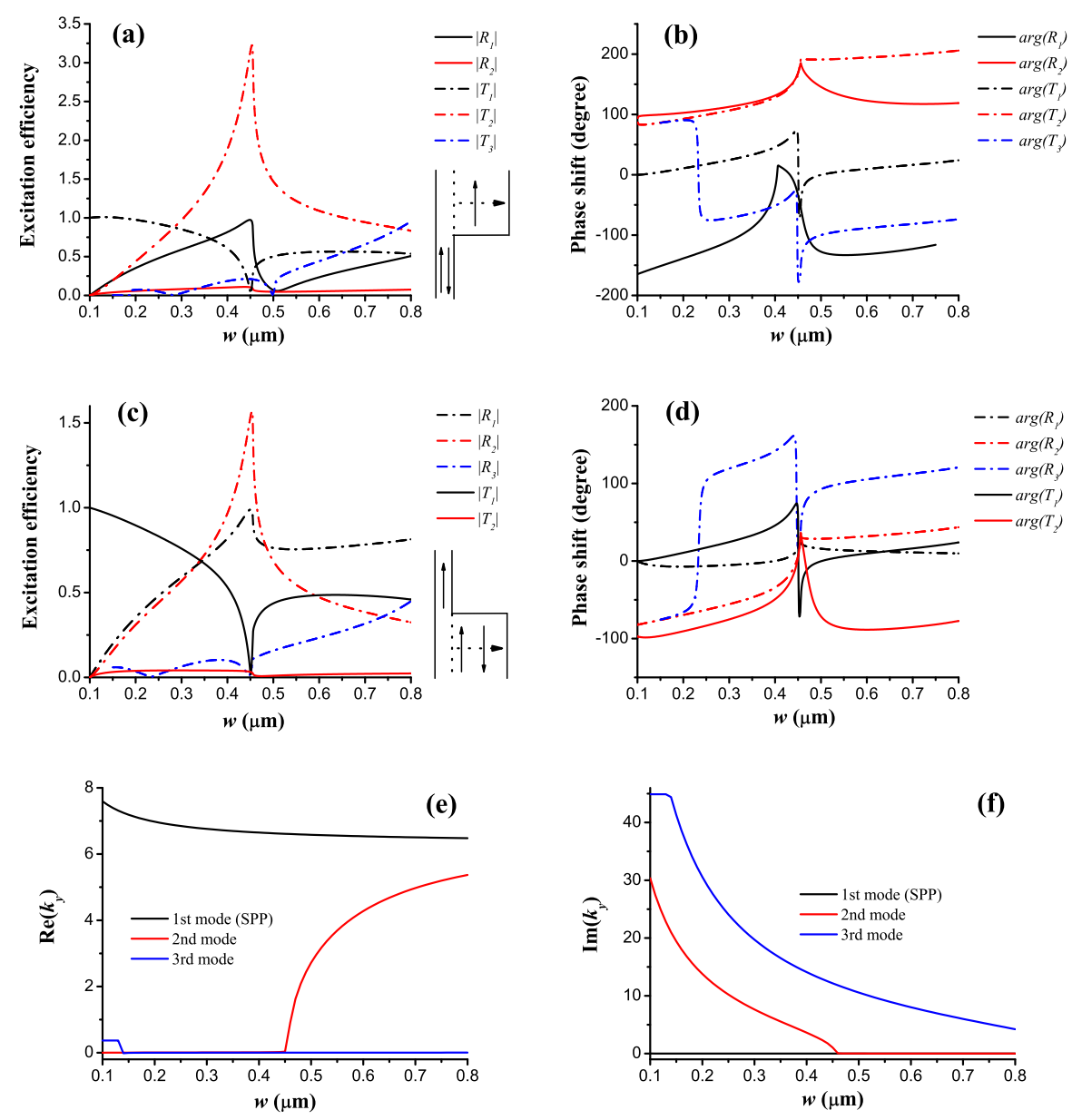

FIG. 3: (color online). Scattering in Type II junction structures. The left walls of the slits are aligned at $x_{1}=0.6 \mu \mathrm{m}$. $Q^{(0)}=Q^{(1)}=Q^{(2)}=0$. In all the figures, the results of the wider slit are plotted by solid lines and those of the narrower slit by dash-dotted lines. The width of the narrower slit is $0.1 \mu \mathrm{m}$. The $x$-axes are the width of the wider slit, denoted as $w$. For wave incident from the narrower slit to the wider one, (a) excitation efficiency; (b) phase shift. For wave incident from the wider slit to the narrower one, (c) excitation efficiency; (d) phase shift. In (e) and (f) plotted are the real and imaginary parts of the propagation constant $k_{y}$, appearing in Eq. (1) with the notation $k_{y}^{(l)}$, of the wider slit. 
When the width of a slit is 0.1 and $0.8 \mu \mathrm{m}$, the first three eigenmodes have been plotted in Figs. 2(e) and (f), respectively. Now the width $w$ varies. Our calculation shows that the modes resemble those in Fig. 2(e) when $w<0.15 \mu \mathrm{m}$ and those in Fig. 2(f) otherwise. The first three eigenmodes are here labeled by the notations $\psi_{1}, \psi_{2}$ and $\psi_{3}$, and their $k_{y}$ 's are by $k_{y 1}, k_{y 2}$ and $k_{y 3}$, respectively.

The SPP mode $\psi_{1}$ is obviously a propagation one since $k_{y 1}$ has a negligible imaginary part, and $\psi_{3}$ is a decaying one as demonstrated by the large imaginary part of $k_{y 3}$. When $w<0.46 \mu \mathrm{m}, k_{y 2}$ is nearly purely imaginary so that $\psi_{2}$ is an evanescent mode, while when $w>0.46 \mu \mathrm{m}, k_{y 2}$ becomes nearly purely real so that $\psi_{2}$ turns to be a propagation mode. A turning point appears at $w=0.46 \mu \mathrm{m}$ at which $\psi_{2}$ transforms its propagation property. This transformation leads to the drastic changes of the excitation efficiencies, similar to the cause of the well-known Wood's anomaly in the grating theory [21].

The analysis about the excitation efficiencies in Figs. 3(a) and (c) are in the same way as those in the Type I structure. Therefore, some similar conclusions are obtained, such as the identity of $\left|T_{1}\right|$ curves in Figs. 3(a) and (c) and the similarity between the efficiencies $\left|R_{1}\right|,\left|T_{2}\right|$ and $\left|T_{3}\right|$ in Fig. 3(a) and $\left|R_{1}\right|,\left|R_{2}\right|$ and $\left|R_{3}\right|$ in Fig. 3(c), respectively, although with different values.

The drastic changes of excitation efficiencies at the turning point have to be explained from an energy perspective. As an example, let us see the case where the wave is incident from the narrower slit to wider one, as shown by the inset in Fig. 3(a). At the start point $w=0.1 \mu \mathrm{m}$, the two slits are the same, so that $\left|T_{1}\right|=1$ and all other excitation efficiencies are zero. Close to the turning point, $\left|T_{1}\right|$ reaches the minimum, and $\left|R_{1}\right|$ and $\left|T_{2}\right|$ reach the maximum. Since the second mode in the wider slit $\psi_{2}$ now is an evanescent mode, the energy is mainly stored in the reflected SPP wave. Once $\psi_{2}$ becomes a propagation mode, it must gain a large portion of energy from the reflection due to its large excitation efficiency, and leads to the rapid drop of $\left|R_{1}\right|$ as shown in Fig. 3(a). At the same time, the dropping $\left|R_{1}\right|$ further causes a redistribution of excitation efficiencies by the boundary continuum condition. Therefore, what behind the drastic changes of excitation efficiencies is a redistribution of energy between evanescent modes and propagation modes. The explanation of the energy redistribution is also suitable for the case where the wave is incident from the wider slit to narrower one.

It is seen from Figs. 3(b) and (d) that the phases of both $T_{1}$ in these two figures are also 
the same. At the turning point, $T_{1}$ changes its phase by $\pi$. The change of $\pi$ in phase at the turning point also occurs for $T_{3}$ in Figs. 3(b) and $R_{3}$ in Figs. 3(d).

So far, the scattering mechanisms of the two types of structures are investigated. Although the investigation here is restricted to the incident wave with wavelength $\lambda_{0}=1 \mu \mathrm{m}$ only, the analysis above is also applicable to the infrared and visible spectrum. Furthermore, the analysis is important in practical application. For example, one can excite/suppress specific modes to control the field distribution inside a slit, or design high efficient reflector, by changing the position or width of the slit.

\section{B. The multi-mode multi-reflection model}

Having had the knowledge of the scattering of the interface in a single junction structure, we are ready in this subsection to discuss the transmission in a step-modulated slit which can be regarded as the combination of two junction structures. In order to reveal the transmission clearly, we present here an analysis of multi-mode multi-reflection model that combines wave and ray optics.

Figure 4 is the sketch of the multi-mode multi-reflection model. In a step-modulated subwavelength metal slit, there are two interfaces at $Q^{(1)}$ and $Q^{(2)}$. Let us discuss the wave reflection and transmission in the slit. When the incident SPP launched from $Q^{(0)}$ in Layer 1 impinges the interface between Layers 1 and $2, Q^{(1)}$, it generates the reflection wave in Layer 1 and transmission wave in Layer 2. The latter continues going upwards, and when reaching other interface $Q^{(2)}$, yields reflection and transmission waves again. Obviously, there occurs multi-reflection in Layer 2, shown by Fig. 4(a).

Because the wave in each layer is the linear combination of the eigenmodes of the layer, each ray in Fig. 4(a) can in fact be expanded by eigenmodes, except the primary incident light. For example, at the point A, the reflected wave contains all possible modes in Layer 1 and the transmitted wave contains the modes in Layer 2. That is to say, the scattering excites all the modes in both layers. When all the possible modes in Layer 2 reach point $\mathrm{B}$, each mode again excites all possible eigenmodes in reflected wave in Layer 2 and in transmitted wave in Layer 3. The phenomenon is termed as multi-mode excitation. To show the phenomenon explicitly, we draw in Fig. 4(b) the multi-mode excitation at point C. Suppose that the waves in Layers 1 and 2 are expanded by three eigenmodes, respectively. 

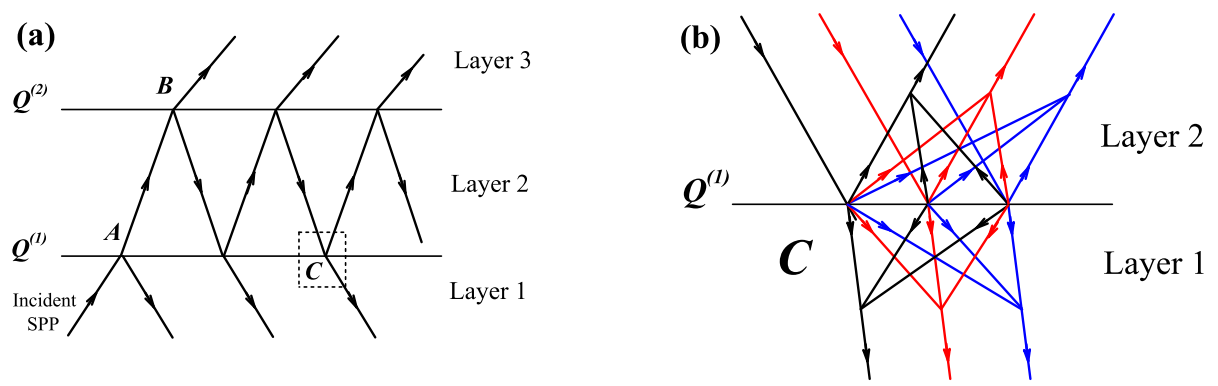

FIG. 4: (color online). Sketch of the multi-mode multi-reflection model. (a) multi-reflection between interfaces and (b) multi-mode excitations at each point.

Then when the three modes in Layer 2 are incident to point C, as shown in Fig. 4(b), the first mode yields the reflected and transmitted waves, both containing three eigenmodes in respective layer, i.e., the incident black line excites the black, red and blue lines in the transmitted waves in Layer 1 and reflected waves in Layer 2, respectively. In the same way, the incident red line also excites the black, red and blue lines in the transmitted waves in Layers 1 and reflected waves in Layer 2, respectively, and so does the incident blue line. Therefore, the total reflected wave at point $\mathrm{C}$ includes three eigenmodes in Layer 2, each being in turn the superposition of the reflections from the three incident eigenmodes. Similarly, the total transmitted wave at point $\mathrm{C}$ includes three eigenmodes in Layer 1, each being in turn the superposition of the transmissions from the three incident eigenmodes.

In summary, the total transmission and reflection coefficients in Layer 3 and Layer 1 in Fig. 4(a) are obtained by summing up all the single-scattered coefficients, respectively. In addition, it is worth mentioning that the multi-mode multi-reflection model is a generalized form of the single-mode multi-reflection model which occurs in a F-P cavity. The former will be simplified to be the latter if only one mode can be excited.

The physical explanation of the multi-mode multi-reflection process is named as model analysis. In order to testify this analysis, numerical calculation based on this physical picture is carried out and the results are compared with MEM. In Fig. 5 plotted are the transmission efficiencies as a function of the length of Layer $2 q^{(2)}$ when the incident wave is SPP mode. The solid lines in Fig. 5(a) and (b) are the results of MEM, which surely comprise the contributions from all possible eigenmodes. The symbols are the results from the model analysis. In a slit with width $w^{(2)}=0.3 \mu \mathrm{m}$, only the first mode, i.e., the SPP mode, can 
propagate and all the other modes are evanescent. Thus, when $q^{(2)}$ is sufficiently long, the higher modes attenuate to a negligible value, and the transmission can be well described by a multi-reflection of only the SPP mode.
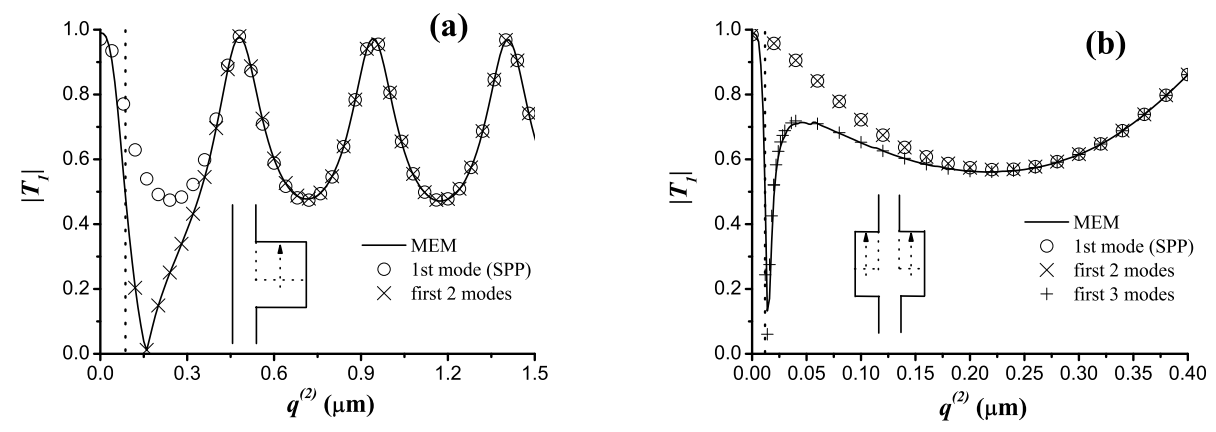

FIG. 5: The SPP transmission vs. $q^{(2)}$ calculated by MEM and model analysis for slit structure with $\left[Q^{(0)}, Q^{(1)}, Q^{(2)}\right]=\left[-1,0, q^{(2)}\right] \mu \mathrm{m}$. The solid lines are the results of MEM. The circles, crosses and plus signs are the results including contributions from the first one, two and three modes, respectively, from the model analysis. (a) Slits align to left at $x_{1}^{(l)}=0.85 \mu \mathrm{m},\left[w^{(1)}, w^{(2)}, w^{(3)}\right]=$ $[0.1,0.3,0.1] \mu \mathrm{m} ;(\mathrm{b})$ The symmetric case of the slit structure in (a).

In Fig. 5(a) it is seen that the results of the model analysis including the first two modes are accurately the same as the line from MEM. When $q^{(2)}>0.8 \mu \mathrm{m}$, the circles and crosses are identical, indicating that the contribution from the second mode is negligible. While for $q^{(2)}<0.8 \mu \mathrm{m}$, crosses deviate from circles, indicating that the second mode should not be omitted since it does not fade out within this distance range. In Fig. 5(a), the crosses end at $q^{(2)}=0.09 \mu \mathrm{m}$, because below this distance the multi-reflection of the first two modes diverges. What is the reason of the divergence? Firstly, the divergence is not caused by the propagation mode since the multi-reflection of the SPP mode always converges, as shown by the circles in Fig. 5(a). Secondly, it is neither caused by the exponentially increasing term which originates from improperly handling the evanescent waves [20] for it occurs only at short distances. Actually, the divergence arises from the coupling between the eigenmodes containing the evanescent modes. With the contribution of the evanescent wave, as shown in Fig. 4(b), the superposition will result in a larger transmission and reflection coefficients after each scattering if the second mode does not decay to a certain value. Thus, there exists a critical distance above which the multi-mode multi-reflection analysis is applicable. For the structure given in Fig. 5(a), it is $q^{(2)}=0.09 \mu \mathrm{m}$. 
To verify the above conclusion about the divergence of the evanescent mode, we suppress the antisymmetric second mode by reforming the slit structure to a symmetric one, as shown in Fig. 5(b). Then the circles and crosses are identical at any distance. Let us see the contribution from the third mode. The plus signs including contributions from the first three modes are in good agreement with the results of MEM as $q^{(2)}>0.012 \mu \mathrm{m}$. The crosses and circles are identical when $q^{(2)}$ is above $0.3 \mu \mathrm{m}$, but it is not so when $q^{(2)}$ is below 0.3 $\mu \mathrm{m}$. That is to say, if the distance is less than $0.3 \mu \mathrm{m}$, the evanescent third mode is not negligible. This time the critical height for the third mode is $q^{(2)}=0.012 \mu \mathrm{m}$, which is much smaller than that of the second mode in the structure shown in Fig. 5(a). The reason is that the decay of the third mode is faster than that of the second mode, for the propagation constant $k_{y}$ of the former has a larger imaginary part than the latter, as shown in Fig. 3(f).
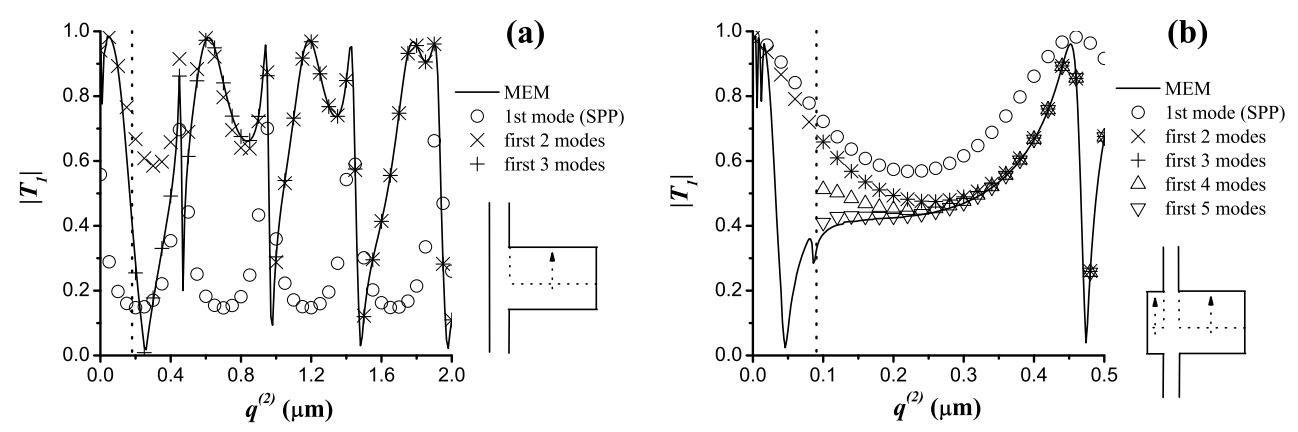

FIG. 6: The SPP transmission vs. $q^{(2)}$ calculated by MEM and model analysis for slit structure with $\left[Q^{(0)}, Q^{(1)}, Q^{(2)}\right]=\left[-1,0, q^{(2)}\right] \mu \mathrm{m}$. The solid lines are the results of MEM. The circles, crosses, plus signs, up-triangles and down-triangles are the results including contributions from the first one to five modes, respectively, from the model analysis. (a) Slits align to left at $x_{1}^{(l)}=0.6$ $\mu \mathrm{m},\left[w^{(1)}, w^{(2)}, w^{(3)}\right]=[0.1,0.8,0.1] \mu \mathrm{m}$; (b) The same structure but the narrower slits are shifted to be $x_{1}^{(1)}=x_{1}^{(3)}=0.744 \mu \mathrm{m}$, a position which totally suppresses the excitation of the 3rd mode, see Fig. 2(a).

Next, we investigate the coupling between propagation modes. In Fig. 5(a), only the first mode, the SPP mode, is the propagation one in Layer 2 with width $w^{(2)}=0.3 \mu \mathrm{m}$. When the width is enlarged, the second mode can also become propagating. In Fig. 6(a) plotted are the transmission efficiencies in the same structures as in Fig. 5(a) except that the width of Layer 2 is extended to be $0.8 \mu \mathrm{m}$. Under this width, the second mode is indeed propagating. It is seen that even $q^{(2)}$ gets to zero, the result containing the contributions 
from the first two modes is not divergent, and agrees with the MEM curve very well when $q^{(2)}>1.2 \mu \mathrm{m}$, which confirm the statement that the propagation modes do not cause the divergence. When $q^{(2)}$ is below $1.2 \mu \mathrm{m}$, the contribution from the third mode has to be added in order to achieve precise results. However, as the cost of preciseness, the divergence appears below $q^{(2)}=0.181 \mu \mathrm{m}$.

Similar to the treatment in Fig. 5(b) where the second mode in Layer 2 is removed by structural change, it is also possible to suppress the third mode excited in Layer 2. The way to implement the suppression is to shift the center of the narrower slits to $x=0.794 \mu \mathrm{m}$, as shown in the inset of Fig. 6(b). At this position, the excitation efficiency of the third mode is nearly zero, see, Fig. 2(a). The transmission results are plotted in Fig. 6(b). It is seen from the figure that up to the first five eigenmodes have to be included in the model analysis in order to meet the MEM curve. The divergence in this case is caused by the fourth mode, and the corresponding critical width is $q^{(2)}=0.084 \mu \mathrm{m}$.

In summary, the multi-mode multi-reflection model provides intuitive and precise description about the transmission inside a step-modulated subwavelength metal slit when the height of modulated layer (Layer 2) is above a critical height, while fails below it because of the coupling between propagation modes and evanescent modes.

\section{Comparison of different methods}

In this subsection, MEM and other three methods, FDTD, SMM, and ICIM, are discussed, and the calculated results of FDTD and ICIM are compared to the MEM results. The preciseness of these methods is investigated and some useful conclusions are obtained. Before presenting the numerical results, we would like to make a brief discussion about these four methods.

FDTD, as a commonly used simulation method in optics, is to calculate field quantities directly from the Maxwell's equations by difference method. In principle, this method and MEM both can provide accurate and reliable results. Here we would like to point out their three discrepancies. Firstly, the way they solve the Maxwell's equations is different: FDTD uses finite difference method to evolve fields in space and time domains, while the MEM establishes and solves the coupled equations in frequency domain by the method of moments. Secondly, the way they handle outmost boundaries is different: FDTD makes 
use of, for the outmost boundaries of a system, perfectly matched layers which can totally absorb waves without reflecting them back, while MEM confines the structure with two perfectly conducting walls such as in this paper. The feasibility of the latter is due to the fast attenuation of light (infrared, visible spectrum) in a metal. If the confined width is large enough, the effects brought by the two perfectly conducting walls are negligible, as shown in Fig 7(a) below. Although the perfectly matched layer technique can be introduced to MEM [22], it dramatically complicates the modal analysis. Thirdly, the way they converge is different: the convergence of FDTD depends on the size of the Yee cell used in simulation, while that of MEM on confined width $L$ and truncation number $N$.

SMM [7-9] and ICIM [13,14] are other two frequently used methods which show following three features. Firstly, according to the two methods, the modulated region, Layer 2, would be divided into a central scattering region and a stub (as shown in the Fig. 2 in Ref. [7] and Fig. 4 in Ref. [13]), and it would assume that the SPP mode multi-reflection occurred in the stub (although Refs. [13] and [14] did not mention this point, it could be recognized from the transmission equations, Eq. (4) in Ref. [13] and Eq. (8) in Ref. [14]). Secondly, both of them took the one mode approximation, which meant that only the SPP modes existed in the stub and slits. Thirdly, the phase shifts caused by scattering in the central scattering region could not be calculated properly, so that were ignored by means of the quasi-statistic approximation $[23,13,14]$ (although Refs. [7-9] did not mention the quasistatistic approximation, it was easily seen by the procedure of obtaining scattering matrix given in Ref. [8]).

In the following, the numerical comparison between these methods is performed. The convergence comparison of MEM and FDTD in a Type I structure is presented in Fig. 7.

Figure 7(a) shows the calculated results of MEM with confined width being $L=2,4 \mu \mathrm{m}$ and truncation number being $N=400,800$, respectively. The results by squares, crosses and plus signs in Fig. 7(a) are identical, showing that the boundary effect imposed by the perfectly conducting walls can be completely ignored in such confined widths. As already mentioned at the beginning of Sec. 3, the parameters $L=2 \mu \mathrm{m}$ and $N=800$ ensure that all the calculated results have at least four significant digits. A FDTD simulated transmission curve with the cell size being $2.5 \times 2.5 \mathrm{~nm}^{2}$ is also plotted in Fig. 7 (a) for comparison. Obviously, the FDTD curve is close to the MEM ones but not coincide. In order to investigate the convergence of FDTD, three simulated transmission curves with 

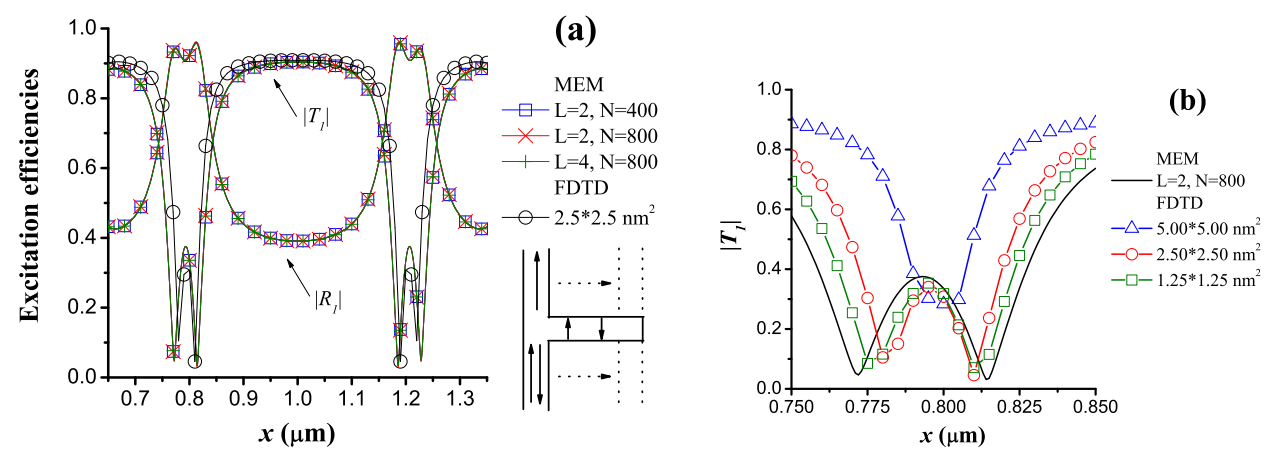

FIG. 7: (color online). The SPP transmission and reflection in a Type I structure with parameter $x_{1}^{(2)}=0.6 \mu \mathrm{m},\left[w^{(1)}, w^{(2)}, w^{(3)}\right]=[0.1,0.8,0.1] \mu \mathrm{m},\left[Q^{(0)}, Q^{(1)}, Q^{(2)}\right]=[-1,0,0.1] \mu \mathrm{m}$, see the inset of (a). (a) Convergence test for MEM; (b) convergence test for FDTD relative to MEM where $x$-axis is a part of (a) for $x_{1}^{(1)} \in[0.7,0.8] \mu \mathrm{m}$.

different cell sizes are plotted in Fig. 7(b) for the same structure as in Fig. 7(a) but with horizontal abscissa being $x_{1}^{(1)} \in[0.7,0.8] \mu \mathrm{m}$ to highlight two absorption peaks. A MEM curve also plotted in the figure for comparison. For large cell size as $5 \times 5 \mathrm{~nm}^{2}$, only one vague dip, instead of two, is observed. The dip will gradually separate into two and approach to the MEM curve as the cell size decreases. However, even for $1.25 \times 1.25 \mathrm{~nm}^{2}$, namely, $1 / 800$ of the incident wavelength or 1/80 of the stub width (height of Layer 2 ), the deviation of the results between FDTD and MEM is still observable, which means that FDTD has a relatively slow convergence. That is why we do not use FDTD to verify the calculated results of MEM in this paper.

The transmission of the structure considered in Fig. 7 was also investigated by SMM [9]. This method actually utilizes some results of FDTD to obtain scatting matrix elements and loses some phase information by quasi-statistic approximation, so that its final results could not be better than that of FDTD. This can be recognized by the comparison between the results of FDTD and SMM given in Refs. [8] and [9]. Therefore, it is not necessary to discuss the preciseness of SMM here because it depends on the simulation results of FDTD which has already shown in Fig. 7(b). Besides, since the SMM and ICIM have the similar transmission model, their calculation errors ought to have the same order of magnitude. The calculation error of ICIM is investigated in the following.

The SPP transmission calculated by ICIM and MEM in a Type II structure are plotted 
in Figs. 8 and 9. This kind of structure was also study in Refs. [13] and [14].
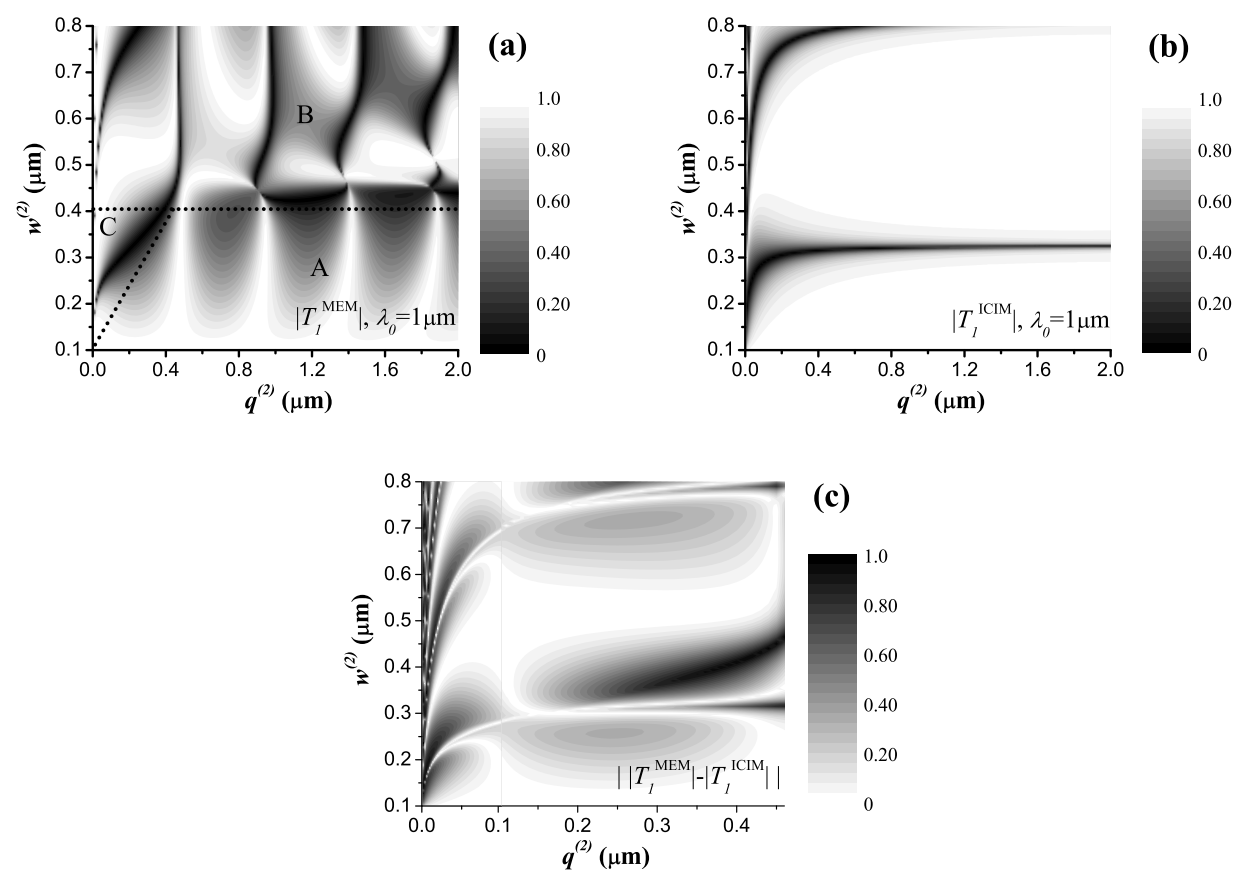

FIG. 8: Comparison of the SPP transmission by MEM and ICIM of a Type II structure under the variation of $w^{(2)}$ and $q^{(2)}$. The left sides of the slits in all layers are aligned to $x_{1}^{(l)}=0.6$ $\mu \mathrm{m} . \quad\left[w^{(1)}, w^{(2)}, w^{(3)}\right]=\left[0.1, w^{(2)}, 0.1\right] \mu \mathrm{m},\left[Q^{(0)}, Q^{(1)}, Q^{(2)}\right]=\left[-1,0, q^{(2)}\right] \mu \mathrm{m} . \quad$ (a) $\left|T_{1}^{M E M}\right|$ by MEM; (b) $\left|T_{1}^{I C I M}\right|$ by ICIM; (c) absolute value of the difference between MEM and ICIM, ||$T_{1}^{M E M}|-| T_{1}^{I C I M}||$ for $q^{(2)}<0.46 \mu \mathrm{m}$.

In Fig. 8 are given the SPP transmission as a function of the height and width of Layer 2 under a fixed incident wavelength, $\lambda_{0}=1 \mu \mathrm{m}$. In Fig. 8(a), the calculated results of MEM can be approximately divided into three areas A, B, and C by dotted lines. In Area A, a regular oscillating pattern is observed because when $w^{(2)}<0.4 \mu \mathrm{m}$ only one SPP mode propagates in Layer 2 that forms the FP-like oscillation $[5,6]$. While in Areas B and C where the higher modes also contribute to transmission, the transmission pattern becomes complicated. Intuitively, there are two ways for higher modes to transport energy. One is in a way of a propagation mode, which is appropriate for $w^{(2)}>0.46 \mu \mathrm{m}$ because the $2 \mathrm{nd}$ mode in Layer 2 becomes propagating. The other is in a way of an evanescent mode, which is appropriate for $0.4<w^{(2)}<0.46 \mu \mathrm{m}$ and Area $\mathrm{C}$ because the 2 nd mode in Layer 2 does not attenuate to a negligible value in such a modulated slit and brings energy through Layer 
The results from ICIM shown in Fig. 8(b) can be discussed according to if $q^{(2)}$ is larger or less than $0.46 \mu \mathrm{m}$. When $q^{(2)}>0.46 \mu \mathrm{m}$, more than one mode are allowed to propagate in the stub, while the ICIM assumes only the SPP mode, which raises the great difference relative to the MEM results, leading to totally different patterns between Figs. 8(a) and (b). When $q^{(2)}<0.46 \mu \mathrm{m}$, the difference ||$T_{1}^{M E M}|-| T_{1}^{I C I M}||$ is plotted in Fig. 8(c). In this region, the difference is mainly caused by the neglect of the phase shifts in the central scattering region, which indicates that these phase shifts have to be taken into account in calculation. In Fig. 8(c), it is seen that when the length of Layer $2 q^{(2)}<0.03 \mu \mathrm{m}$, less than $1 / 30$ of the incident wavelength, the difference becomes larger due to the energy transported by evanescent modes. This demonstrates that a very narrow region cannot guarantee precise results. Thus, the application of the quasi-statistic approximation in a modulated metal slit requires an optimum geometry in order to provide relatively accurate results: the incident wavelength is nearly 10 times larger than stub width.

In order to test the optimum geometry, the slit width of Layer 2 and the incident wavelength $\lambda_{0}$ are considered as variables and the length of Layer 2 is set as $q^{(2)}=0.1 \mu \mathrm{m}$, the same as the slit widths in Layers 1 and 3. The MEM results in Fig. 9(a) exhibit a few straight black bands indicating the inverse-proportional relation between frequency and stub width which was mentioned in Refs. [8] and [9]. For ICIM, similar results are obtained in Fig. 9(b). The difference of these two figures is plotted in Fig. 9(c). Clearly, the calculation error of ICIM in this case is lower than that in Fig. 8(c). Although small deviation takes place near the absorption peaks, ICIM successfully predicts the positions of the peaks. All these numerical results confirm that the ICIM can provide relatively accurate results when the incident wavelength is around 10 times of the stub width.

Anyway, each method has its own advantages in certain aspect. For example, FDTD can provide visualized transmission process in time domain and ICIM is indubitably the fastest method in calculation. Here we just emphasize that these methods should be used with caution in considering the convergence and preciseness. 

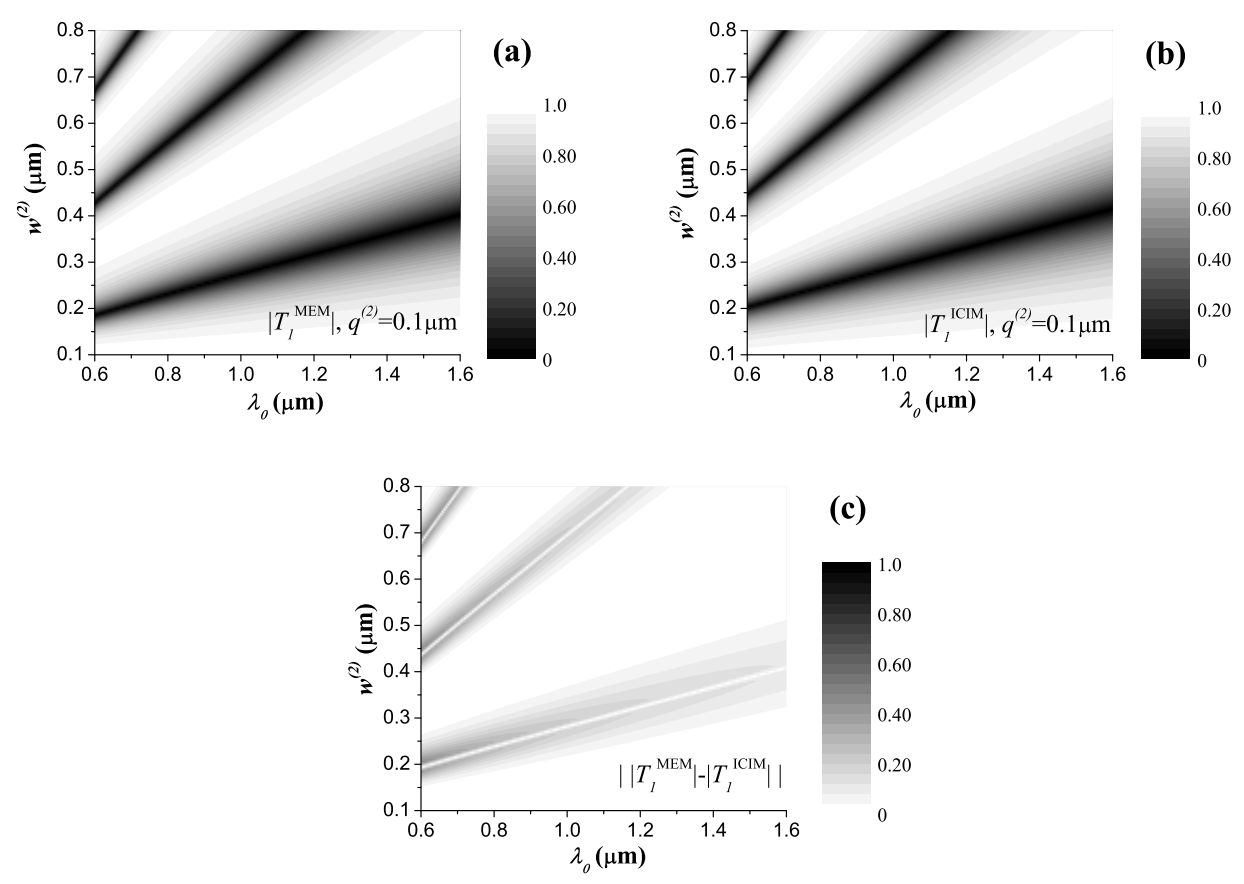

FIG. 9: Comparison of the SPP transmission by MEM and ICIM of a Type II structure under the variation of $w^{(2)}$ and wavelength $\lambda_{0}$. The left sides of the slits in all layers are aligned to $x_{1}^{(l)}=0.6 \mu \mathrm{m} . \quad\left[w^{(1)}, w^{(2)}, w^{(3)}\right]=\left[0.1, w^{(2)}, 0.1\right] \mu \mathrm{m}, \quad\left[Q^{(0)}, Q^{(1)}, Q^{(2)}\right]=[-1,0,0.1] \mu \mathrm{m} . \quad$ (a) $\left|T_{1}^{M E M}\right|$ by MEM; (b) $\left|T_{1}^{I C I M}\right|$ by ICIM; (c) absolute value of the difference between MEM and ICIM, ||$T_{1}^{M E M}|-| T_{1}^{I C I M}||$.

\section{CONCLUSION}

In this paper, the MEM developed in Ref. [6] is improved to be a more practical and efficient one for handling the scattering/transmission. Using this method, the scattering in a juncture structure and the transmission inside a step-modulated slit are investigated.

Firstly, the scattering in a juncture structure is studied for two types of structural changes. For the Type I change where the widths of the two slits are fixed and the position of the narrower one can be anywhere within the wider one, the excitation efficiencies of the modes in the wider slit resemble their eigenfunctions respectively, while in the narrower slit the excitation efficiency of the SPP mode is dominant and much larger than that of the second mode. For the Type II change where the left walls of the slits are aligned and one slit becomes wider gradually, a wood-anomaly-like drastic change of excitation efficiencies is observed when the propagation property of one mode begins to transform. 
Then, the transmission inside a step-modulated slit is studied. Besides the MEM calculation, we present explicitly a multi-mode multi-reflection model to reveal the transmission process. The multi-mode excitation and the superposition procedure of the scatterings from all possible modes are the key parts of the model, which represent the interference and energy transfer happened at layer boundaries. However, there exists a critical height of the modulated layer for applying the model due to the coupling between propagation modes and evanescent modes. Above the critical height, the model can provide the same result as MEM.

In addition, some commonly used methods are compared with MEM. Useful conclusions about these methods are obtained: for a subwavelength metal slit, MEM is a versatile and fast method that can provide accurate results; FDTD has a relatively slow convergence and need very small Yee cell to ensure the accuracy of the simulation; ICIM incorporating the one mode and quasi-statistic approximations provides relatively accurate results when the incident wavelength is around 10 times larger than the stub width.

\section{Acknowledgments}

This work is supported by the 973 Program of China (Grant No.2011CB301801) and the National Natural Science Foundation of China (Grant No. 10874124), and (Grant No. $11074145)$.

† E-mail: 263zys@263.net

‡ E-mail: wanghuaiyu@mail.tsinghua.edu.cn

1 W. L. Barnes, A. Dereux and T. W. Ebbesen, Nature (London) 424, 824 (2003).

2 J. A. Dionne, L. A. Sweatlock, and H. A. Atwater, Phys. Rev. B 73, 035407 (2006).

3 B. Sturman, E. Podivilov, and M. Gorkunov, Phys. Rev. B 76, 125104 (2007).

4 A. R. Zakharian, J. V. Moloney, and M. Mansuripur, Opt. Express 15, 183 (2007).

5 C. Li, Y. S. Zhou, H. Y. Wang, and F. H. Wang, J. Opt. Soc. Am. B 27, 59 (2010).

6 C. Li, Y. S. Zhou, H. Y. Wang, and F. H. Wang, Opt. Express 19, 10073 (2011).

7 X. S. Lin and X. G. Huang, Opt. Lett. 33, 2874 (2008). 
8 X. S. Lin and X. G. Huang, J. Opt. Soc. Am. B 26, 1263 (2009).

9 J. Tao, X. G. Huang, X. S. Lin, J. H. Chen, Q. Zhang, and X. P. Jin, J. Opt. Soc. Am. B 27, $323(2010)$.

10 Q. Zhang, X. Huang, X. Lin, J. Tao, and X. Jin, Opt. Express 17, 7549 (2009).

11 A. Hosseini and Y. Massoud, Opt. Express 14, 11318 (2006).

12 Y. S. Zhou, B. Y. Gu, and H. Y. Wang, Phys. Rev. A 81, 015801 (2010)

13 Y. Matsuzaki, T. Okamoto, M. Haraguchi, M. Fukui, and M. Nakagaki, Opt. Express 16, 16314 (2008).

14 A. Pannipitiya, I. D. Rukhlenko, M. Premaratne, H. T. Hattori, and G. P. Agrawal, Opt. Express 18, 6191 (2010).

15 P. B. Johnson and R. W. Christy, Phys. Rev. B 6, 4370 (1972).

16 L. C. Botten, M. S. Craig, and R. C. McPhedran, Computer Phys. Commun. 29, 245 (1983).

17 M. G. Moharam, E. B. Grann, D. A. Pommet, and T. K. Gaylord, J. Opt. Soc. Am. A 12, 1068 (1995).

18 L. Li, J. Opt. Soc. Am. A 13, 1870 (1996).

19 L. Li, J. Opt. Soc. Am. A 13, 1024 (1996).

20 M. G. Moharam, D. A. Pommet, E. B. Grann, and T. K. Gaylord, J. Opt. Soc. Am. A 12, 1077 (1995).

21 A. Hessel and A. A. Oliner, Appl. Opt. 4, 1275 (1965).

22 Q. Cao, P. Lalanne, and J. P. Hugonin, J. Opt. Soc. Am. A 19, 335 (2002).

23 S. Ramo, J. R. Whinnery, and T. Van Duzer, Fields and Waves in Communication Electronics (Wiley, New York, 1994). 